\title{
Low-cost Delivery and Telepresence Robot for COVID-19 Crisis
}

\author{
Arnon Jumlongkul ${ }^{*}$ \\ ${ }^{1}$ School of Medicine, Mae Fah Luang University, Chiang Rai, Thailand \\ * Correspondence: Arnon Jumlongkul \\ arnon.jum@mfu.ac.th
}

Keywords: COVID-19, delivery robot, healthcare robot, telepresence robot, Smart hospital.

\begin{abstract}
Purpose: The ongoing COVID-19 crisis has created the need for social distancing between medical staff and patients, therefore, healthcare robotics is increasingly important as regards infectious control. The purpose of this study was to design and fabricate a remote controlled transport robot that could provide an optimum technology base for developing countries, having dual functions of telepresence and delivery operations inside or outside buildings, is easy to assemble and also easily able to be cleaned using standard chemical agents.

Methods: This machine was designed and fabricated to incorporate two main functions; delivery and telepresence. The robot consists of a base frame, DC motors for driving and steering systems, actuator motor for driving wheel elevation, Wi-Fi AP/Repeater, network camera, and a necessary a back-up power system.
\end{abstract}

Results: This robot can be used both indoors and outdoors. It can carry loads of more than $80 \mathrm{~kg}$. The machine can cross obstacles greater than $3 \mathrm{~cm}$. It can be cleaned by disinfectant agents. An actuator motor can lift the driving wheels above the surface to prevent gear damage.

Conclusion: This article demonstrates the concept of a healthcare telepresence and delivery robot that can provide distancing during the present COVID-19 crisis, using optimum technology for low and middle-income countries. In the future, the researcher should consider development of the delivery controls, combined with the telepresence system using the internet of things, to improve the navigation system, the manipulator system for supporting multi-floor transportation, and also other multifunctional purposes.

\section{Introduction}

To minimize the spread of Covid-19, "social distancing" has become a major necessity. To provide social distancing, the Centers for Disease Control and Prevention (CDC) has recommended everyone to maintain at least 6 feet between themselves and others, to avoid groups and stay out of crowded places [1]. An increasingly used solution for the creation of social distancing is healthcare robotics. Emerging technologies in medical robots have included robots for stock control, cleaning, delivery, sterilization, surgery, telepresence, as companions, cognitive therapy, rehabilitation, and also humanoid robots [2]. For medical logistics tasks, delivery robots have been chosen to help prevent dissemination of COVID-19. Some hospitals have requested automated transport vehicles, which can collect and deliver any materials, are easy to load and unload by healthcare personnel, as well as be programmed for specific locations for pick-up and delivery. Delivery or service robots should be 
intuitive to control, to send an object to a destination, easy to command via touch screens and even detect and avoid obstacles [3].

When considering telecommunication between healthcare personnel and COVID-19 patients, who by necessity must stay in a quarantine ward or negative pressure isolation room, a teleoperated or telepresence robot is needed for this mission. The main concept of telepresence robotic function is to establish a meeting which is not limited only to a conference room, by amalgamating communication with a video-conference system, also called mobile robotic telepresence (MRP) system. MRP systems generally consist of a web camera, amplifier, LCD screen, two-way communication, and ideally also have mobility. In healthcare systems, telepresence robots have been used in many areas, for instance, postoperative care units, intensive care units (ICU), anatomy laboratories, in surgical teaching and elderly in place facilities [4]. Therefore, the emergence of telepresence and delivery robots is an appropriate technology for the present COVID-19 crisis and the 'new normal' era. However, in Thailand, we also lack an optimum prototype of a telepresence system amalgamated with a delivery robot that can provide an economic dual transportation and communication machine to support social distancing in a hospital. Almost all delivery robot prototypes cannot be incorporated with an aluminum cart. Sometimes a mechanical system must be attached to a cart with drilling screws, bolts, or cutting some parts of a cart, then, an existing cart will be useless if the user needs to remove a cart for other applications.

During this COVID-19 pandemic situation, many companies have tried to apply automated solutions to support their healthcare workers against viral exposure, for example, using fixed-based and mobile manipulators to perform tasks, developing wheeled mobile robots, flying mobile robots, legged mobile robots, wearable robots for measurement of body signals, exoskeletal robots for strengthening and rehabilitation, etc. The mechanical designs of human-robot interaction modality have also included telerobotics (e.g., the da Vinci Surgical Robot), collaborative robots (e.g., semiautonomous ultrasound scans), autonomous robots (e.g., UV sterilization robots), wearable robots for measuring body signals and physical activity of patients, and also social robots, like humanoids, respectively. To manage medical infection control tasks, telerobots have inevitably become perceived as leading actors [5]. One of the robotics in global COVID-19 healthcare management is a serving robot, which is deployed to supply food, drugs, beverages, and laundries to COVID-19 patients in many Chinese as well as Singapore hospitals [6]. Due to the rapid outbreak of COVID-19, in China, fourteen robots were deployed in the field hospital named Wuhan Hongshan Stadium. They were used to deliver drugs and food, disinfect, measure patient temperature, and entertain patients. Like in Italy, the Tommy robot is used to communicate to patients visually and also acoustically [7]. However, because of budgetary restraints, lockdown policies, and technology shortfalls, to design and build an intelligent healthcare robot in developing countries within a short period will be an unlikely occurrence. Even though these robots can provide social distancing, by the way, they also need the best Wi-Fi system within a building, which has not been adequate in remote areas, especially in many rural community hospitals in developing countries.

So to summarize, the purpose of this article was to attempt to design and fabricate a healthcare robot for use against COVID-19 that uses an optimum technology for low and middle income countries, to provide dual functions of telepresence and delivery operations, that is easy to assemble, similar to a general hospital cart, easy to clean with standard anti-microbial agents, and that also can support effective telecommunications both inside and outside a hospital building.

\section{Materials and Methods}


Some considerations, already mentioned in this article, were separated into 2 areas as follows:

\subsection{Delivery Function and Circuit Architecture}

The machine concept is centered on usefulness, low-cost, and compatibility with most hospital carts of varying sizes. Therefore, an optimum design of healthcare robotics should be able to be assembled such that existing carts can be easily mounted to and removed from the new compact robot without drilling for screws or bolts. The machinery control parts of the robot were designed using SolidWorks 2017 software. The structural base frame was constructed from steel sprayed with rust-proof color. The upper part of the base frame can be adjusted according to the size of a cart. This robot consists of 4 PVC caster wheels, which can be turned through 360 degrees. The driving and steering systems use dual $24 \mathrm{~V} 150 \mathrm{~W}$ DC motors, driving the pneumatic tired wheels via a worm gear NMRV030, which can drive the left and right wheel independently. The battery system consists of dual $12 \mathrm{~V} 45 \mathrm{Ah}$ Lithium batteries, joined in a series, for both delivery and telepresence functions, the batteries being housed in stainless steel boxes. To prevent driving system damage, a linear $100 \mathrm{~mm} 24 \mathrm{~V}$ DC actuator motor is used to elevate the pair of driving wheels free from the ground when the electric system is off.

The electrical circuit is mainly composed of two sets of voltage protection circuits, which cause cut off when the voltage drops below $23.8 \mathrm{~V}$, and switches on when the voltage is above $23.9 \mathrm{~V}$. The remotecontrol system receiver was designed to support 10 channels. The DC motors are controlled by a pulse width modulation (PWM) system, which receives signals from the remote control, allowing two channels for each driving wheel as well as another set for the linear motor. A step-up boost converter, which can convert the voltage from $24 \mathrm{~V}$ to $48 \mathrm{~V}$, was provided for a fitted surveillance camera. All electronic parts were housed in a waterproof electrical control box. The electrical circuit of the driving system is shown in Figure 1. 


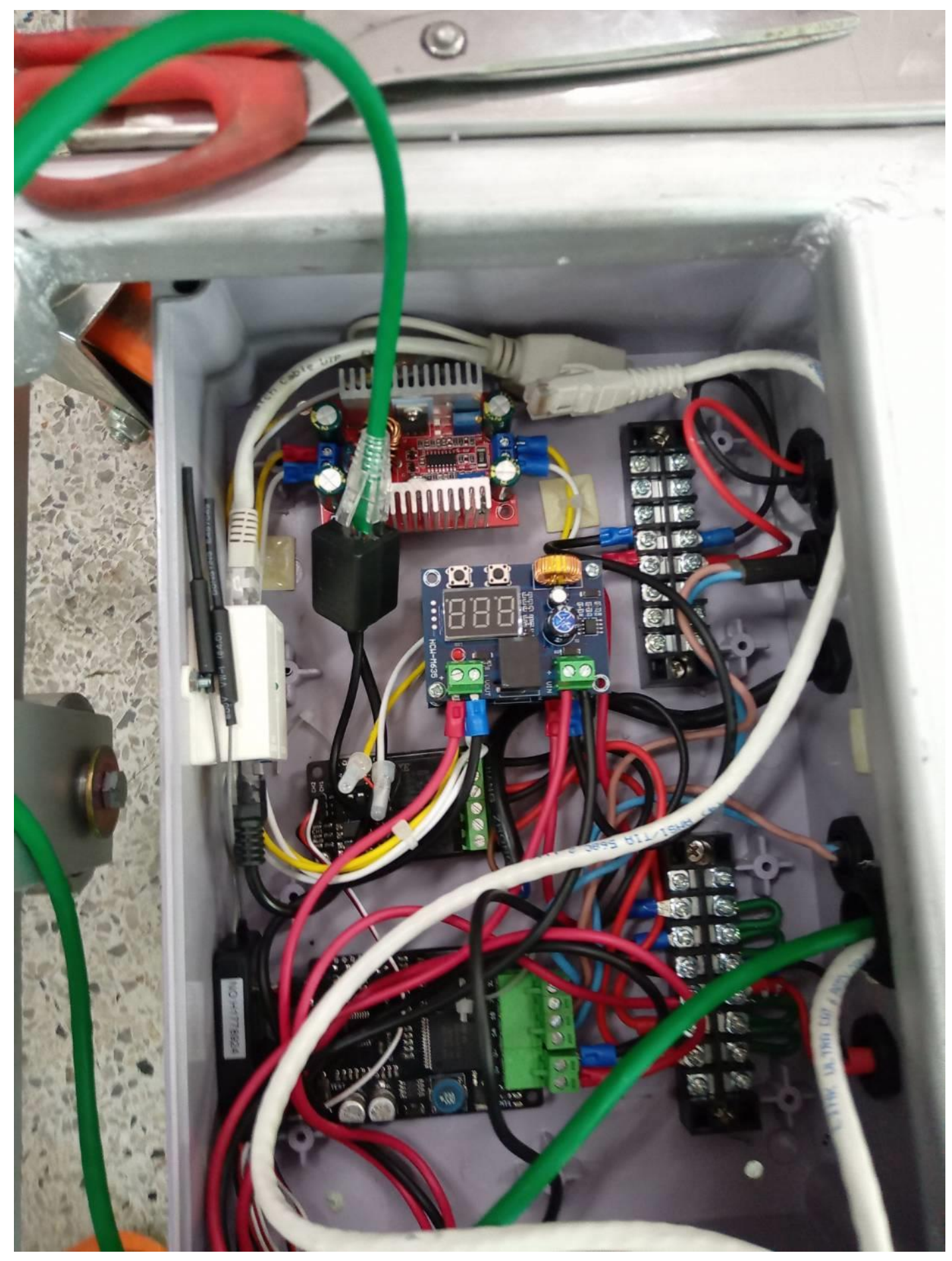

Figure 1. Electrical circuit of the driving system

A waterproof switch box houses two DC voltmeters, with LCD voltage displays, one for each of the functions of transportation and communication. A wireless 6-10 channel $2.4 \mathrm{GHz}$ STN transflective display, powered by $6 \mathrm{~V} \mathrm{DC} 1.5 \mathrm{AA} * 4$ batteries, based on a model airplane remote control, was used for operator control of the delivery system. The entire structure was designed to allow protection of the electronics from disinfectant cleaning solutions. To replicate and build this model, the robot drawing is shown in Figure 2. The dimensions of the delivery robot are shown in Figure 3. 


\subsection{Telepresence Function}

Because of budget limitations many hospitals in developing countries cannot provide a viable Wi-Fi connection in all areas. Sometimes essential infrastructure is not present. So therefore a local network was selected as the first choice to compensate for this situation. A waterproof high-power outdoor WiFi AP/Repeater, which has dual-band speeds of up to $2.4 \mathrm{GHz} 150 \mathrm{Mbps}$ and also $5 \mathrm{GHz} 433 \mathrm{Mbps}$, was used to provide high performance communication for the telepresence system. A network camera, 5 MP IR fixed cube, lens $2.8 \mathrm{~mm}$, was assembled with a Wi-Fi repeater. A local network from telepresence devices were joined with a tablet PC, which was installed with iVMS-4200 client software for two-way communication.

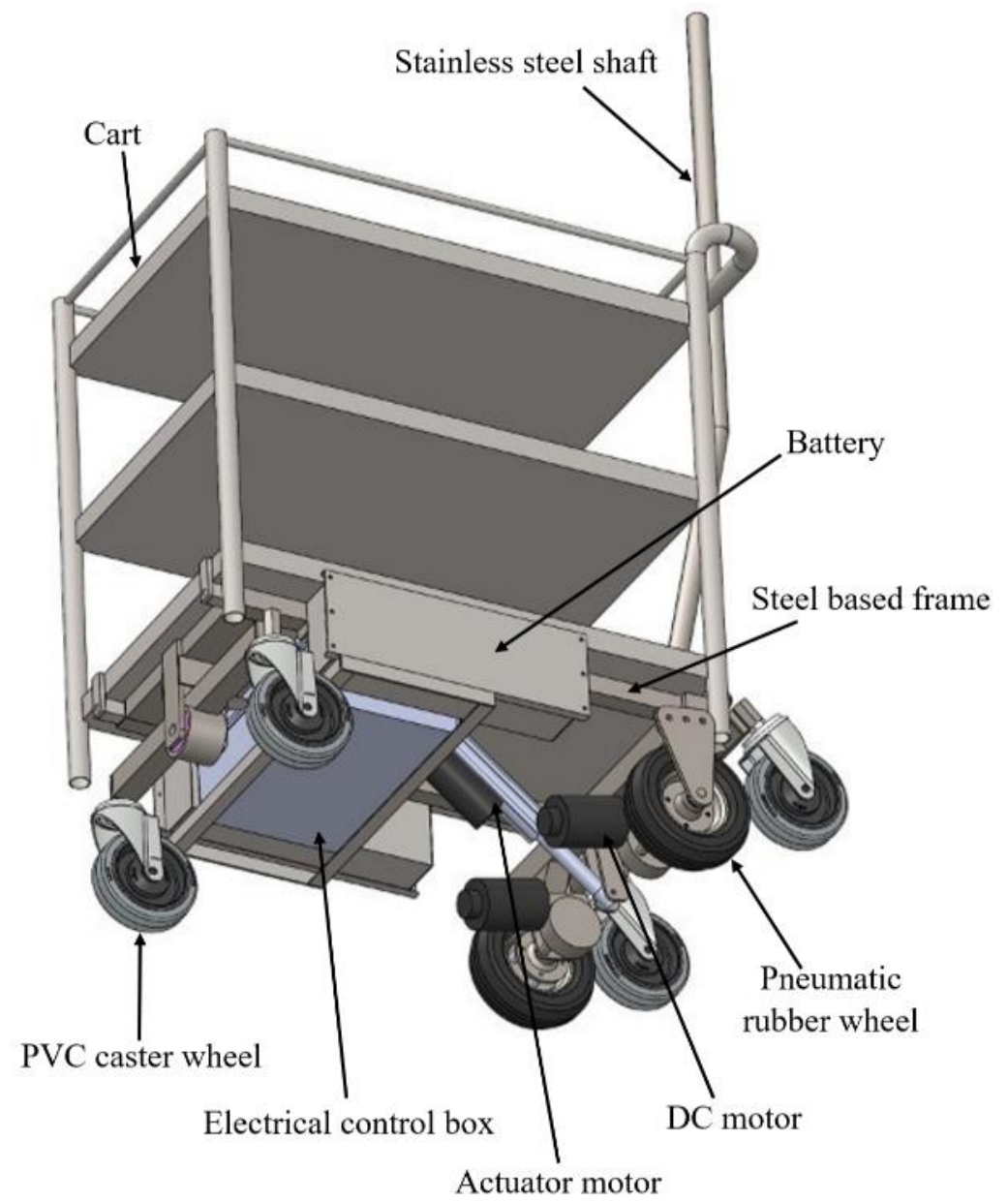

Figure 2. Delivery robot drawing 

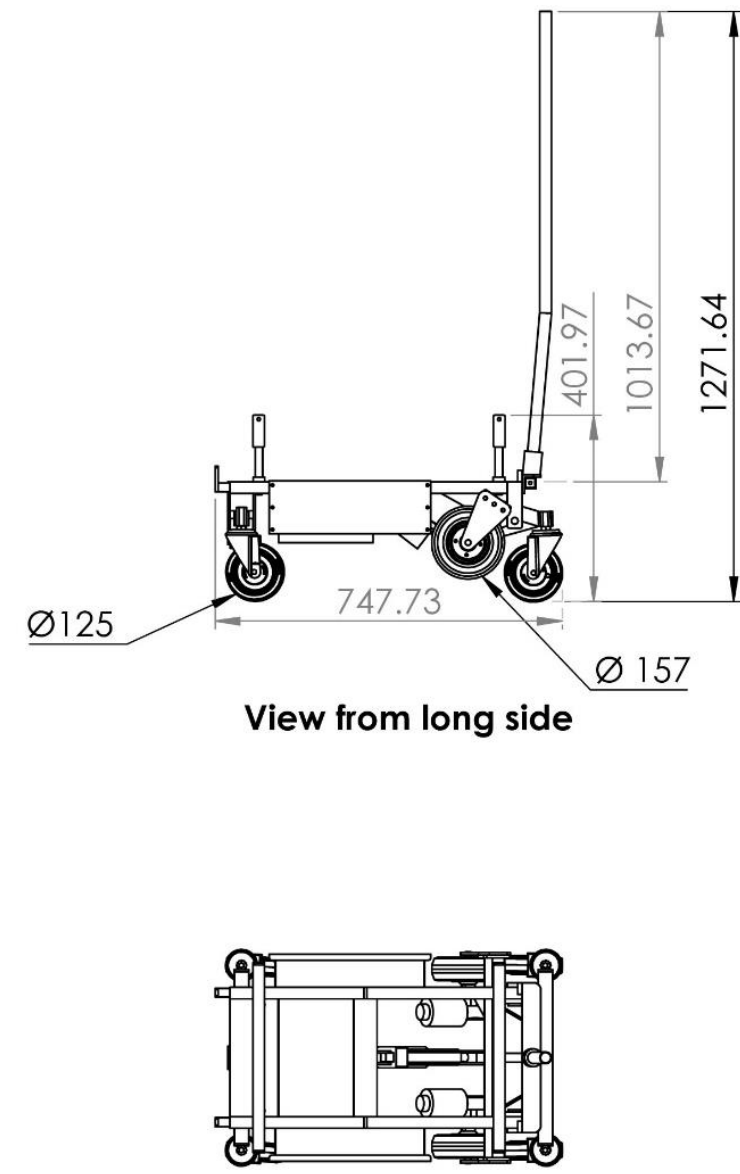

Top view

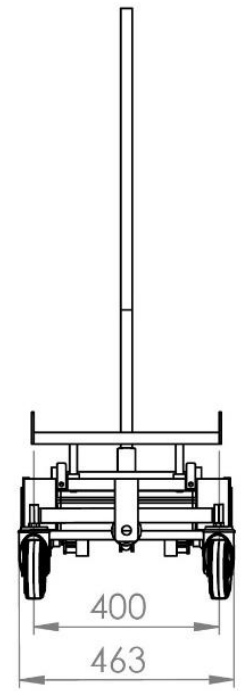

View from wide side

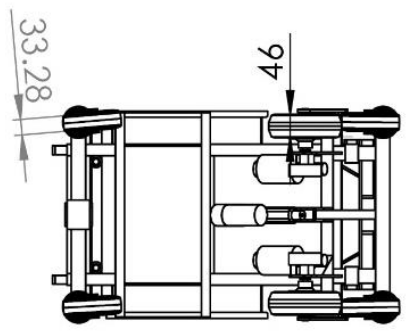

Below view

Figure 3. Dimensions of the delivery robot (millimeter)

\section{$3 \quad$ Results}

The model of delivery and telepresence robot to help manage the ongoing COVID-19 crisis was fabricated and tested. The results are shown below:

\subsection{Control Systems Test}

The operative range, via the remote control, and the wireless camera, exceeded $50 \mathrm{~m}$ within a building and reached $100 \mathrm{~m}$ outdoors. Operators not familiar with this machine will need time to develop control skills because the robot caster wheels can be rotated in any direction. The prototype of this robot is shown in Figure 4 and Figure 5. The test of Wi-Fi connection is shown in Figure 6. The test of delivery system is shown in Figure 7 and Figure 8.

\subsection{Driving and Loading Test}

The actuator motor can lift the driving wheels up to $6 \mathrm{~cm}$ above the surface to prevent gear damage when the user needs to manually move the robot. The speed of the driving system is controlled @ $1 \mathrm{~m}$ $\mathrm{s}^{-1}$ maximum speed. When operating on level ground, this robot, can carry up to $80 \mathrm{~kg}$. The machine can cross a door sill or similar obstacle, measuring more than $3 \mathrm{~cm}$ in height. The robot can be amalgamated with a hospital cart easily by simply putting the cart on the top of the robot base frame. 
Because of the dual functions of the driving and steering systems, using the two-wheel drive, the robot needs only a little space for making a U-turn. When the user moves the remote-control button to let the robot run forward while turning left, then, the left wheel will slow down while the right wheel will be ongoing. If the machine operator orders the robot to turn right, without making a forward motion, the left wheel will turn steadily while the right wheel will be stationary.

\subsection{Disinfectant test}

According to the concern about COVID-19 persists, the robot was tested with any disinfectant sprays and water. Results showed that cleaning agents as well as water cannot make contact with or damage the electrical devices which are located within the base frame. However, to prevent the long term electronics damage, rubbing the machine dry is recommended.

\subsection{Cost-effectiveness}

The total cost of this machine that was calculated in Thai Baht (THB) is 111,500 THB. To discover the appropriate technique, some materials were bought with the uselessness of their applications. Therefore, to replicate this robot after the model is finalized, the budgets may decline approximately $30 \%$. 


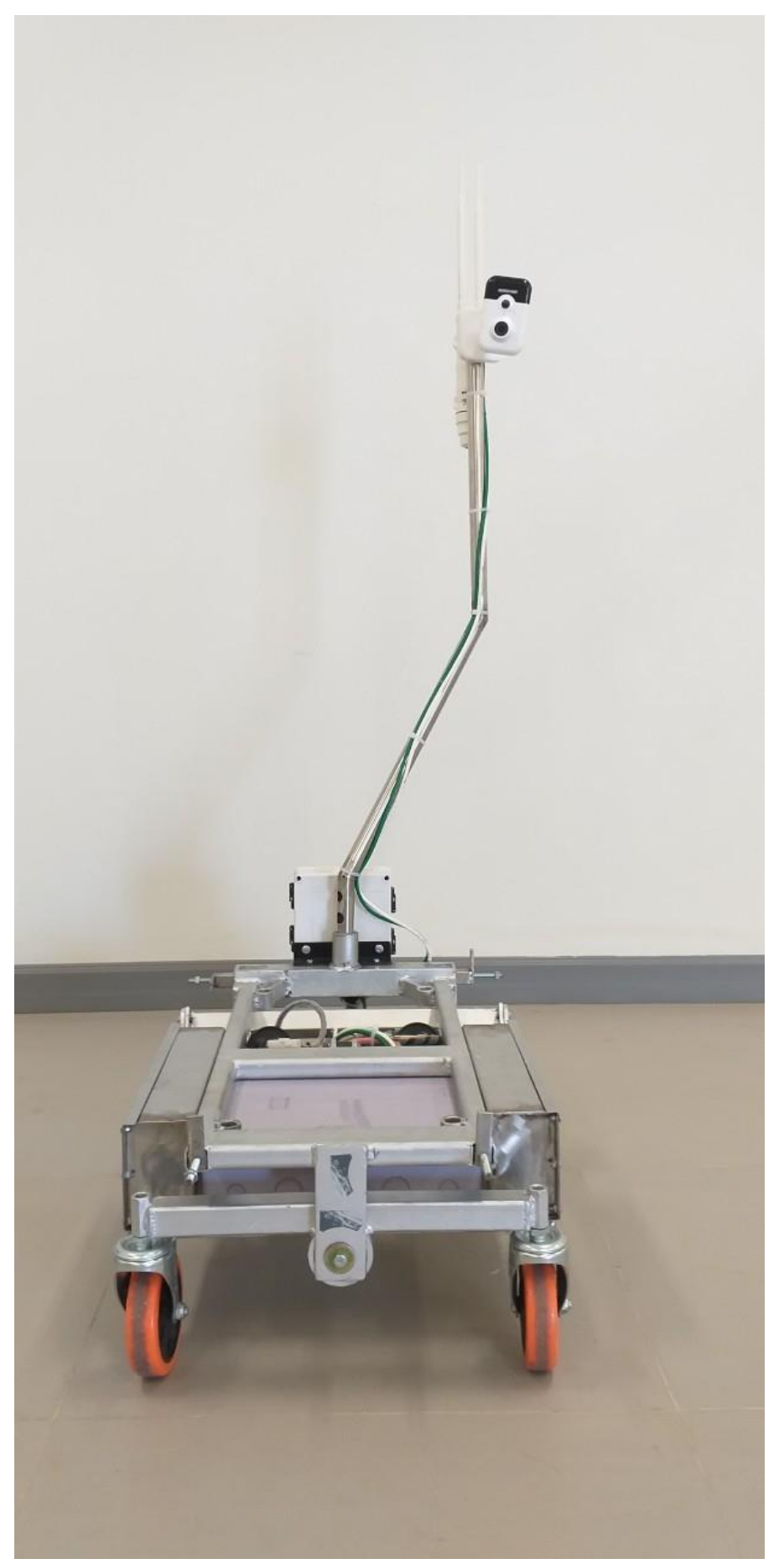

Figure 4. Delivery and telepresence robot (front view) 


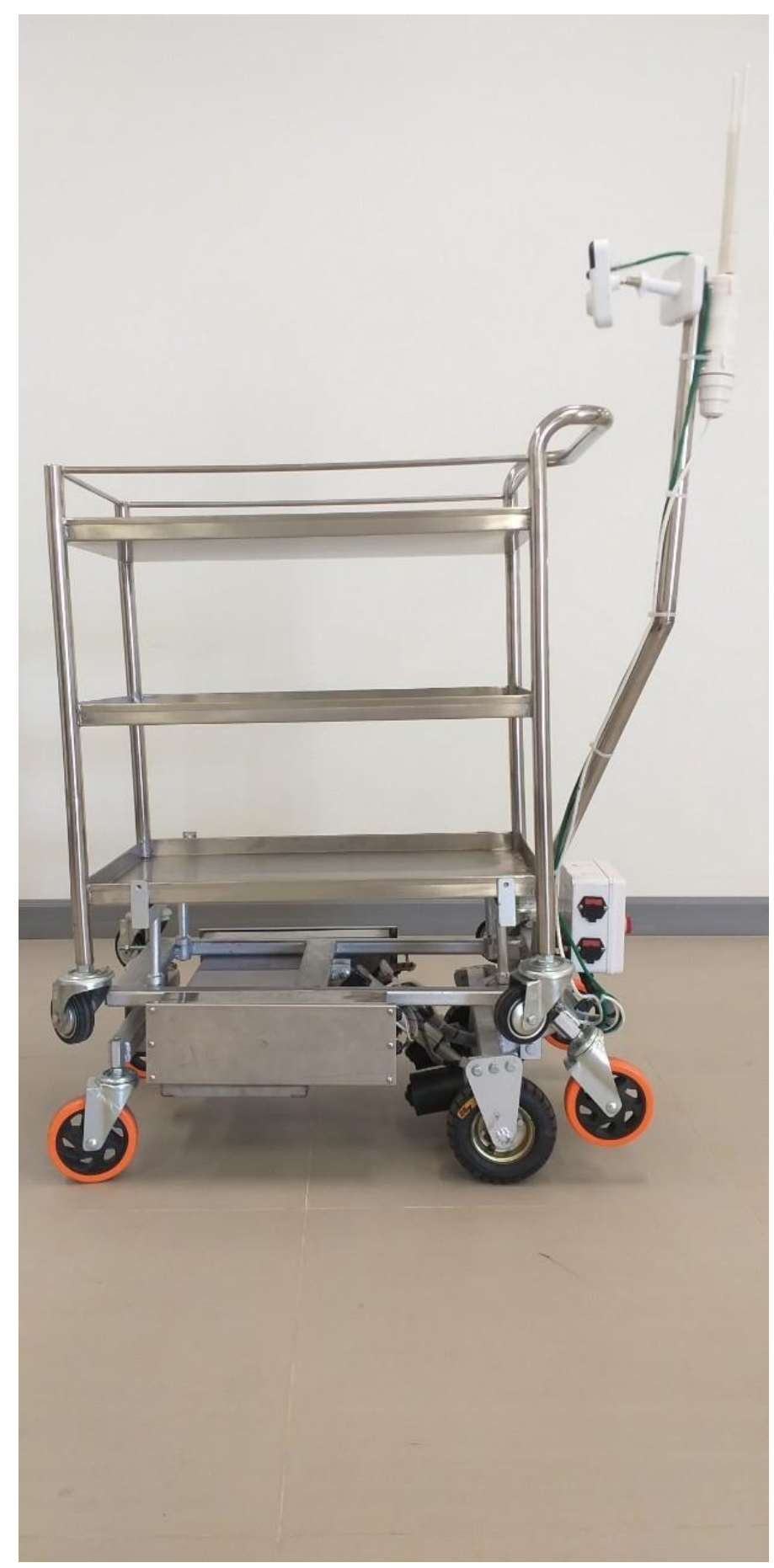

Figure 5. Delivery and telepresence robot with cart (side view) 


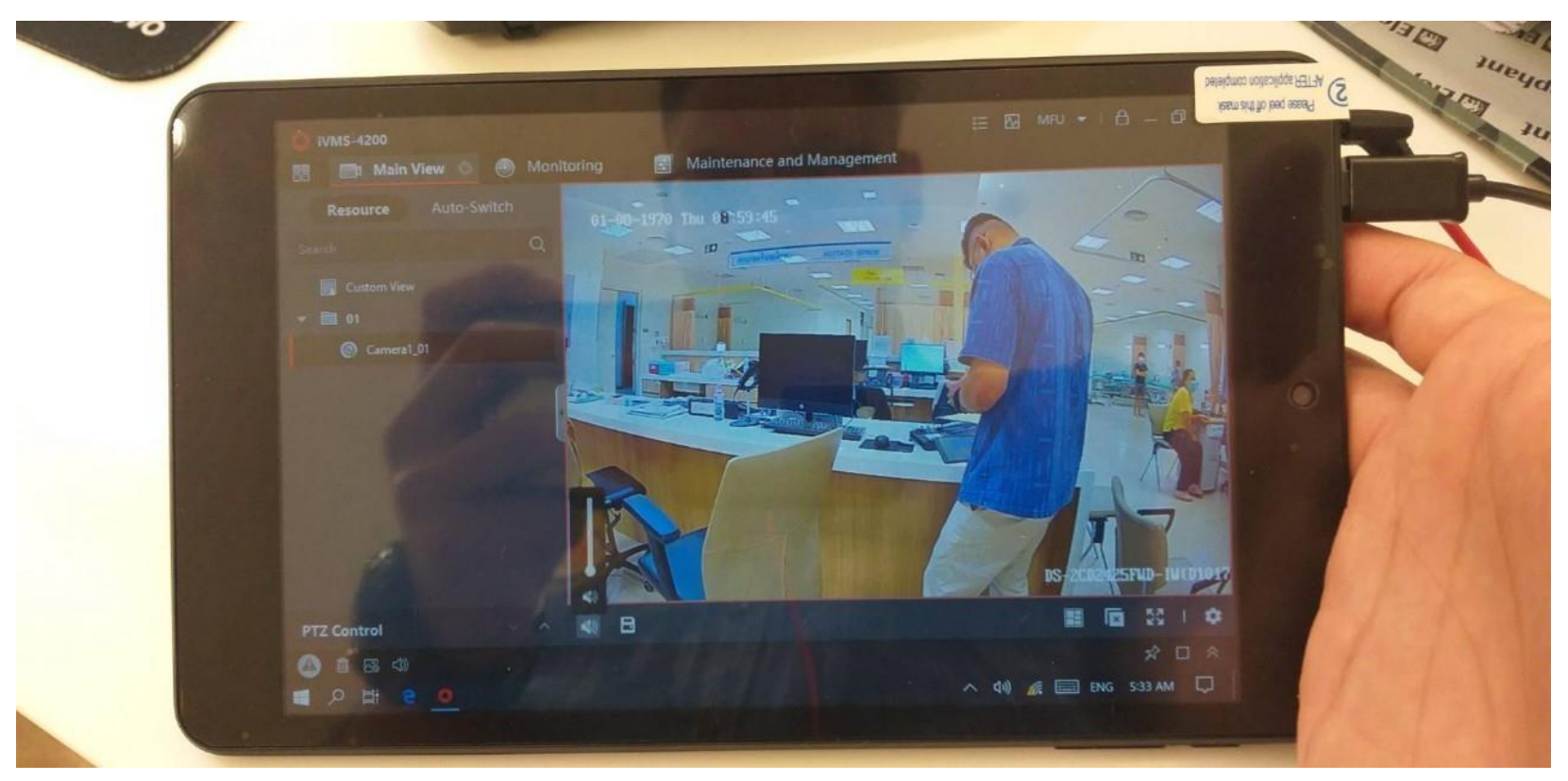

Figure 6. Wi-Fi connection between a robot camera and a tablet PC within an emergency room

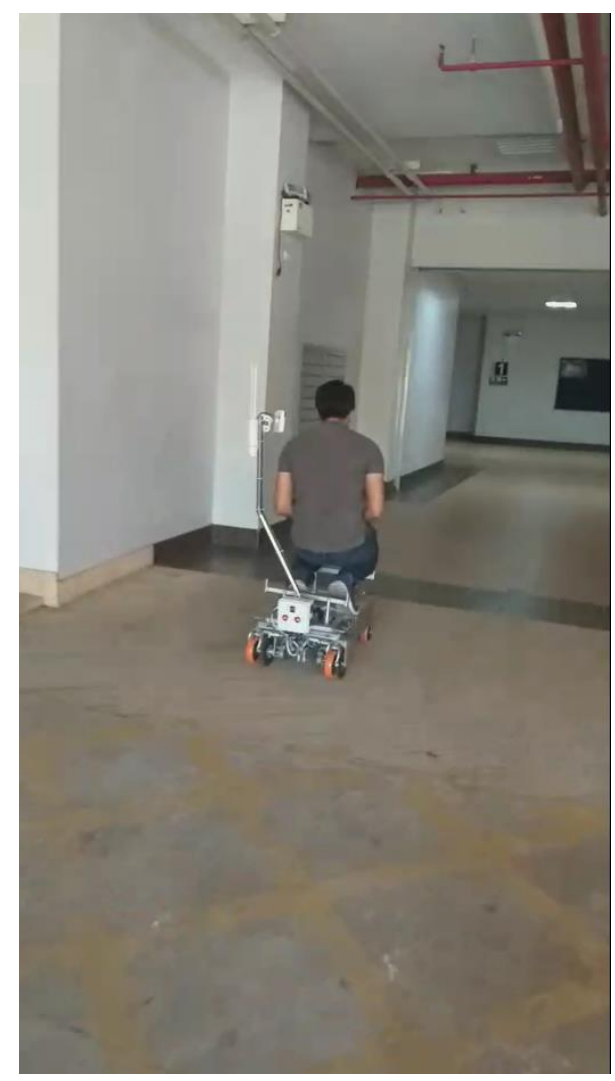

Figure 7. The test of the delivery function when loaded an $85 \mathrm{~kg}$ man 


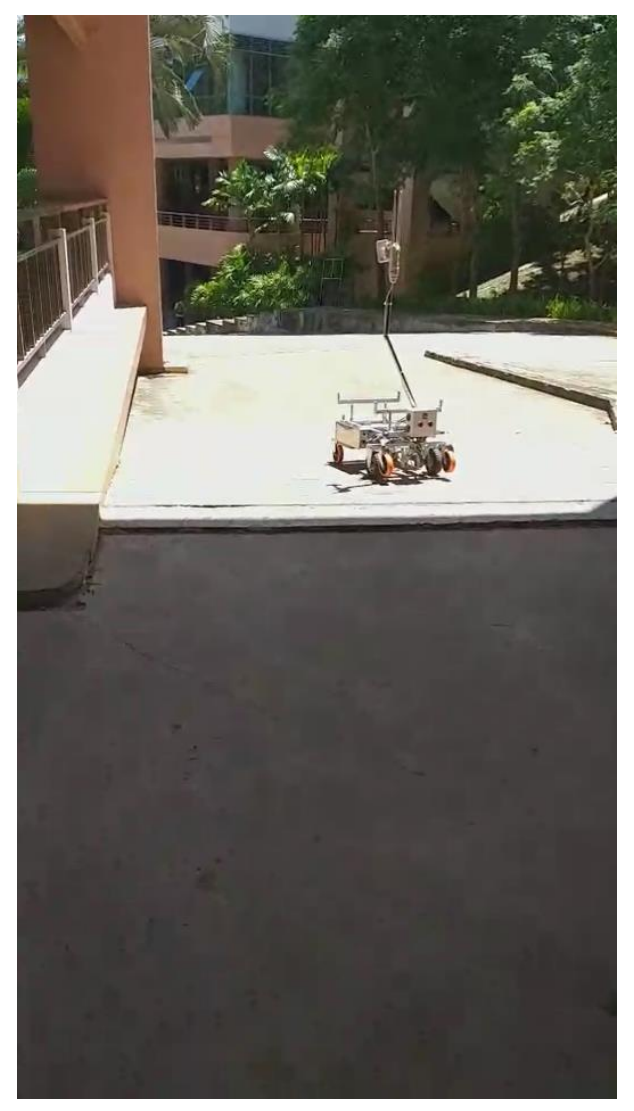

Figure 8. The test of the driving system when operating on level ground, measuring $30^{\circ}$ to $45^{\circ}$ in declination angle

\section{Discussion}

This delivery and telepresence robot was fabricated within the limitation of time as well as monetary resources. The machine has been used in the Mae Fah Luang University Medical Center Hospital, Chiang Rai Province, which is far away from Bangkok and also lack of raw materials. At this time, this robot is ready to support COVID-19 mitigation strategies, mostly limited to rooms within an inpatient faculty. However, now we have no COVID-19 case that has been detected within our healthcare service areas. Therefore, this machine has been used and tested in an emergency room. If we have time and budgets enough, for greater functionality, some technical details need to be addressed. On this version the telepresence system and delivery system are independent of each other so the user must control both devices simultaneously. A way to solve this problem would be to connect any robot control functions via a mobile application, which could provide robot control interaction using voice or touch screen in an all in one tablet. Because of incomplete obstacle detection and navigation systems, which were fabricated in a short time, the user must personally check for obstacles as the wireless camera has no obstacle detection function. A similar product in Turkey showed implementation of real time obstacle detection in a robotic cart using an android application, Arduino platform, that has a buzzer for proximity warning, a passive infrared sensor (PIR) to detect humans, an ultrasonic sensor to calculate the distances of objects or obstructions, and also makes use of Bluetooth technology [8]. Ongoing research is in the development of autonomous navigating technology, which has been applied to a robotic vehicle for use in dangerous environments. This version has many functions, for example, navigation, mapping, and manipulation. The main structure of this autonomous 
robot also incorporates depth cameras, laser scanners, manipulators, and also inertial measurement sensors [9]. These technologies may be merged with internet of things technology for the future versions.

Secondly, our present machine can be operated only either on the same floor or in open outdoor environs, although it could be moved to other floors using an elevator. For a genuine multi-floor transportation system, a mobile robot should be programmed with a multi-floor navigation system (e.g., mapping, path planning, and localization), elevator control system (3D camera and ultrasonic sensors), labware manipulation technology, which would be combined with an arm control system, and also a collision avoidance system $[10,11]$.

Finally, according to the axiomatic design (AD) theory, the trend of development of innovative medical devices requires a multi-functional conceptual design for delivering medical supplies. Therefore, the future healthcare delivery and telepresence robot should support varied delivery tasks, for example, drugs, organs, fluids, surgical instruments, etc. Also a future medical robot should be developed that can control systems related to cooling, heating, humidifying, and also dehumidifying, respectively [12].

\section{Conclusions}

This paper reveals the design and fabrication of a healthcare remote controlled transport robot, incorporating dual telepresence and delivery functions, to help mitigate the COVID-19 crisis. The functional concept required an optimum technology that can be available to low and middle-income countries. Medical personnel can assemble this machine easily by putting a cart onto the transport robot, the base frame of which can be adjusted according to the size of the cart. Waterproof and stainless-steel boxes can protect all machinery and electronic parts from corrosive agents, which would be used for robot cleaning. The present telecommunication system is suitable for use in both indoor and outdoor environments. According to the trial results, future research should consider development of the delivery control system becoming amalgamated with the telepresence system via internet of things technology. The navigation system being able to detect obstacles and then linking to a mapping recognition software. The manipulator technology being able to support multi-floor transportation via an elevator, and finally a multi-functional robot being able to assist with some medical tasks.

\section{Conflict of Interest}

The author has no conflict of interest to declare.

\section{$7 \quad$ Author Contributions}

Arnon Jumlongkul created this robot as well as drafted the manuscript solely.

\section{$8 \quad$ Funding}

This research is supported by the Princess Srinagarindra's Centenary Celebrations Foundation, Mae Fah Luang University, fiscal year 2020, Memorandum No. 28/2563.

\section{Acknowledgments}

The inventor would like to thank Dr. Roger Timothy Callaghan, School of Medicine, Mae Fah Luang University for linguistic analysis, Watchara Jamnuch as well as Thanat Sirsuksan, an electrical engineer, for technical information. 


\section{References}

1. Centers for Disease Control and Prevention. Social Distancing [Internet]. 2020 [cited 2020 May 18]. Available from: https://www.cdc.gov/coronavirus/2019-ncov/prevent-getting-sick/socialdistancing.html

2. Cresswell K, Cunningham-Burley S, Sheikh A. Health Care Robotics: Qualitative Exploration of Key Challenges and Future Directions. J Med Internet Res. 2018 04;20(7):e10410.

3. Acosta Calderon CA, Mohan ER, Ng BS. Development of a hospital mobile platform for logistics tasks. Digit Commun Netw. 2015 Apr;1(2):102-11.

4. Kristoffersson A, Coradeschi S, Loutfi A. A Review of Mobile Robotic Telepresence. Adv HumComput Interact. 2013;2013:1-17.

5. Tavakoli M, Carriere J, Torabi A. Robotics, Smart Wearable Technologies, and Autonomous Intelligent Systems for Healthcare During the COVID-19 Pandemic: An Analysis of the State of the Art and Future Vision. Adv Intell Syst. 2020 May 5;2000071.

6. Khan ZH, Siddique A, Lee CW. Robotics Utilization for Healthcare Digitization in Global COVID19 Management. Int J Environ Res Public Health. 2020 May 28;17(11):3819.

7. Zeng Z, Chen P-J, Lew AA. From high-touch to high-tech: COVID-19 drives robotics adoption. Tour Geogr. 2020 May 26;22(3):724-34.

8. Y1lmaz E, Özyer ST. Remote and Autonomous Controlled Robotic Car based on Arduino with Real Time Obstacle Detection and Avoidance. Univers J Eng Sci. 2019 Jan;7(1):1-7.

9. Losch R, Grehl S, Donner M, Buhl C, Jung B. Design of an Autonomous Robot for Mapping, Navigation, and Manipulation in Underground Mines. In: 2018 IEEE/RSJ International Conference on Intelligent Robots and Systems (IROS) [Internet]. Madrid: IEEE; 2018 [cited 2020 Jul 10]. p. 140712. Available from: https://ieeexplore.ieee.org/document/8594190/

10.Thurow K, Zhang L, Liu H, Junginger S, Stoll N, Huang J. Multi-floor laboratory transportation technologies based on intelligent mobile robots. Transp Saf Environ. 2019 Jul 1;1(1):37-53.

11.Abdulla AA, Liu H, Stoll N, Thurow K. A New Robust Method for Mobile Robot Multifloor Navigation in Distributed Life Science Laboratories. J Control Sci Eng. 2016;2016:1-17.

12.Zhu A, Zou C, Luo W, He R. The Demand-driven Conceptual Design of Multi-function Modular Cabinet for Medical Delivery Robot. Procedia CIRP. 2016;53:273-7. 